\title{
How should we teach cardiopulmonary resuscitation? Randomized multi-center study
}

\author{
Burak Katipoglu ${ }^{1}$, Marcin Andrzej Madziala ${ }^{2}$, Togay Evrin ${ }^{1}$, Pawel Gawlowski ${ }^{3}$, \\ Agnieszka Szarpak ${ }^{4}$, Agata Dabrowska ${ }^{5}$, Szymon Bialka ${ }^{6}$, Jerzy Robert Ladny ${ }^{7}$, \\ Lukasz Szarpak ${ }^{2}$, Anna Konert ${ }^{4}$, Jacek Smereka ${ }^{3}$ \\ ${ }^{1}$ Department of Emergency Medicine, Ufuk University Medical Faculty, \\ Dr. Ridvan Ege Education and Research Hospital, Cankaya, Ankara, Turkey \\ ${ }^{2}$ Medical Simulation Center, Lazarski University, Warsaw, Poland \\ ${ }^{3}$ Department of Emergency Medical Service, Wroclaw Medical University, Wroclaw, Poland \\ ${ }^{4}$ Lazarski University, Warsaw, Poland \\ ${ }^{5}$ Department of Rescue Medical Service, Poznan University of Medical Sciences, Poznan, Poland \\ ${ }^{6}$ Department of Anesthesiology and Intensive Care, Medical University of Silesia, Katowice, Poland \\ ${ }^{7}$ Department of Emergency Medicine and Disaster, Medical University Bialystok, Poland
}

\begin{abstract}
Background: A 2017 update of the resuscitation guideline indicated the use of cardiopulmonary resuscitation (CPR) feedback devices as a resuscitation teaching method. The aim of the study was to compare the influence of two techniques of CPR teaching on the quality of resuscitation performed by medical students.

Methods: The study was designed as a prospective, randomized, simulation study and involved 115 first year students of medicine. The participants underwent a basic life support (BLS) course based on the American Heart Association guidelines, with the first group (experimental group) performing chest compressions to observe, in real-time, chest compression parameters indicated by software included in the simulator, and the second group (control group) performing compressions without this possibility. After a 10-minute resuscitation, the participants had a 30-minute break and then a 2-minute cycle of $C P R$. One month after the training, study participants performed CPR, without the possibility of observing real-time measurements regarding quality of chest compression.

Results: One month after the training, depth of chest compressions in the experimental and control group was $50 \mathrm{~mm}$ (IQR 46-54) vs. $39 \mathrm{~mm}$ (IQR 35-42; $p=0.001$ ), compression rate 116 CPM (IQR 102-125) vs. 124 CPM (IQR 116-134; $p=0.034)$, chest relaxation 86\% (IQR 68-89) vs. 74\% (IQR $47-80 ; p=0.031$ ) respectively.

Conclusions: Observing real-time chest compression quality parameters during BLS training may improve the quality of chest compression one month after the training including correct hand positioning, compressions depth and rate compliance. (Cardiol J 2021; 28, 3: 439-445)
\end{abstract}

Key words: basic life support, learning, medial simulation, quality, chest compression

\section{Introduction}

Out-of-hospital cardiac arrest is a global health problem, with survival varying greatly between communities. Sudden cardiac arrest (SCA) is one of the leading causes of death in Europe. Depending how SCA is defined, 55-113 per 100,000 inhabitants per year or 350,000-700,000 individuals each year are affected in Europe [1,2]. On initial heartrhythm analysis, $25-50 \%$ of SCA victims have ventricular fibrillation (VF), a percentage that has declined over the last 20 years [3, 4]. However,

Address for correspondence: Marcin Andrzej Madziala, PhD, Medical Simulation Center, Lazarski University, ul. Świeradowska 43, 02-662 Warszawa, Poland, tel: +48 519160 829, e-mail: rat.poz@wp.pl

Received: 29.05.2019 Accepted: 20.08.2019

This article is available in open access under Creative Common Attribution-Non-Commercial-No Derivatives 4.0 International (CC BY-NC-ND 4.0) license, allowing to download articles and share them with others as long as they credit the authors and the publisher, but without permission to change them in any way or use them commercially. 
regardless of the rhythm initiating cardiac arrest, the key is to implement resuscitation procedures as soon as possible [5].

The guidelines of the European Resuscitation Council (ERC) as well as the American Heart Association (AHA) indicate the need for high quality chest compression as an element closely correlated with the efficiency of cardiopulmonary resuscitation (CPR). Both the ERC and AHA guidelines provide a detailed description of how chest compression should be performed.

One of the key elements of the recent emphasis has been on minimizing chest compression interruptions [6]. According to Ewy et al. [7] the most optimal form of chest compression is continuous compression, which generates higher perfusion pressure than resuscitation based on 30 compressions to 2 rescue breaths. To this purpose, it may be essential to perform airway management with an endotracheal tube or supraglottic airway device and initiate asynchronous resuscitation, so that chest compression interruptions, necessary for ventilation with a face mask and a self-inflating bag, are minimized [8-10]. Further parameters indicated by the guidelines include the depth and the rate of compressions as well as the correctness of chest relaxation after each compression. However, regardless of whether resuscitation is based on European or American guidelines, as numerous studies indicate, the quality of chest compressions performed even by medical staff is insufficient $[8,11-14]$.

The 2017 update of the resuscitation guideline indicated the use of CPR feedback devices [15] as a resuscitation teaching method. Numerous studies indicate that chest compression using these devices is superior to standard resuscitation [16-18]. However, because of the relatively high cost of these devices they are encountered sporadically during real-life resuscitation activities as well as during training courses. It is therefore crucial to seek new methods of teaching both basic and advanced resuscitation procedures which will improve the performance of chest compressions.

The aim of the study was to compare the influence of two techniques of CPR teaching on the quality of resuscitation performed by medical students.

\section{Methods}

\section{Study design}

The study was designed as a prospective, randomized, simulation study. The study protocol was approved by the Institutional Review Board (IRB) of the Polish Society of Disaster Medicine
(Approval no.: 24.11.2017.IRB). Following IRB approval and written informed consent, 115 first year students of medicine took part in the study.

\section{Study protocol}

To simulate a patient with cardiac arrest requiring CPR, Resusci Anne ${ }^{\circledR}$ QCPR (Laerdal, Stavanger, Norway) was used, which was placed on a flat surface in a brightly lit room.

Before starting the study, the participants were divided into two groups and ResearchRandomizer (randomizer.org) was used for this purpose. In both groups a 5 minutes standardized training on how to perform CPR of an adult was performed prior to the study. Both groups then underwent a basic life support (BLS) course based on the AHA guidelines, with the first group (experimental group) performed chest compressions to observe, in real-time, chest compressions parameters indicated by software included in the simulator, and the second group (control group) performed compressions without the possibility of observing simulator indications. After a 10 -minute resuscitation, the participants had a 30-minute break and then a 2-minute cycle of CPR based on a scheme of 30 compressions: 2 rescue breaths. The first group performed compressions on the basis of simulator indications, while the second group did not.

The next phase of the study was conducted 1 month after training. At that time study participants in the same groups performed CPR, this time both experimental and control groups were not able to observe real-time measurements regarding quality of chest compression.

\section{Measurements}

During the study, parameters of chest compression were analyzed, including total compression score, calculated by simulator software on the basis of parameters of chest compression. Additionally, compression depth, compression depth compliance, compression rate per minute (CPM), compression rate compliance, full release as well as correctness of chest position during compression were evaluated. As reference values for depth and rates of chest compressions, the values recommended by the AHA were used, this states that the optimal depth of adult chest compressions is between 50 and $60 \mathrm{~mm}$ and the optimal rate of compressions should be between 100 and 120 CPM [19]. All chest compression parameters were recorded by dedicated software included in the SkillReporter (Laerdal, Stavanger, Norway). 
Table 1. Chest compression (CC) data.

\begin{tabular}{lccc}
\hline Parameter & $\begin{array}{c}\text { Control group } \\
\text { Manual CC } \\
\text { (n = 56) }\end{array}$ & $\begin{array}{c}\text { Experimental group } \\
\text { The device feedback } \\
\text { (n = 55) }\end{array}$ & P \\
\hline Before practical training & & & \\
Total compression score [\%] & $70(43-82)$ & $69(41-80)$ & NS \\
Compression depth [mm] & $39(37-42)$ & $39(36-42)$ & NS \\
Compression depth compliance [\%] & $68(54-74)$ & $69(52-75)$ & NS \\
Compression rate [per min] & $128(116-131)$ & $124(114-130)$ & NS \\
Compression rate compliance [\%] & $70(51-83)$ & $71(50-84)$ & NS \\
Full release [\%] & $76(53-85)$ & $77(55-84)$ & NS \\
Correct hand position [\%] & $83(71-90)$ & $83(70-92)$ & NS \\
After training & & & \\
Total compression score [\%] & $74(51-85)$ & $93(87-100)$ & $\mathbf{0 . 0 0 1}$ \\
Compression depth [mm] & $40(39-44)$ & $51(48-57)$ & $<\mathbf{0 . 0 0 1}$ \\
Compression depth compliance [\%] & $68(60-89)$ & $96(90-100)$ & $\mathbf{0 . 0 0 1}$ \\
Compression rate [per min] & $124(110-128)$ & $110(103-121)$ & $\mathbf{0 . 0 1 9}$ \\
Compression rate compliance [\%] & $78(54-88)$ & $97(92-100)$ & $\mathbf{0 . 0 0 1}$ \\
Full release [\%] & $76(53-90)$ & $91(81-97)$ & $\mathbf{0 . 0 3 7}$ \\
Correct hand position [\%] & $83(76-94)$ & $96(92-100)$ & $\mathbf{0 . 0 0 7}$ \\
One month after training & & & \\
Total compression score [\%] & $74(50-79)$ & $90(84-100)$ & $<\mathbf{0 . 0 0 1}$ \\
Compression depth [mm] & $39(35-42)$ & $50(46-54)$ & $\mathbf{0 . 0 0 1}$ \\
Compression depth compliance [\%] & $64(50-71)$ & $94(90-100)$ & $<\mathbf{0 . 0 0 1}$ \\
Compression rate [per min] & $124(116-134)$ & $116(102-125)$ & $\mathbf{0 . 0 3 4}$ \\
Compression rate compliance [\%] & $72(53-74)$ & $97(89-100)$ & $\mathbf{0 . 0 0 1}$ \\
Full release [\%] & $74(47-80)$ & $86(68-89)$ & $\mathbf{0 . 0 3 1}$ \\
Correct hand position [\%] & $80(70-91)$ & $94(81-100)$ & $\mathbf{0 . 0 1 7}$ \\
\hline N & & & \\
\hline
\end{tabular}

NS - not statistically significant

\section{Statistical analysis}

Data were analyzed with the use of Statistica software v.13.3EN (TIBCO., Tulsa, OK). The results are shown as medians and interquartile ranges (IQR). The occurrence of normal distribution was confirmed by the Kolmogorov-Smirnov test. Analysis of variance (ANOVA) post hoc tests with the Bonferroni correction for metric data were used for univariate analysis to compare the two study groups. The Kruskal-Wallis test was used to compare non-normally distributed data. Multivariate ANOVA was also applied. The results were considered significant at the level of $\mathrm{p}<0.05$.

\section{Results}

One hundred and fifteen students in their first year of medical studies were enrolled in the study, however, in the initial phase of the study 4 persons decided not to participate in the study. Randomization took place for 111 participants.

A detailed summary of data obtained in the study is presented in Table 1 . The initial chest quality assessment performed before the training did not show statistically significant differences between the experimental group and the control group.

After training, study participants had access to a monitor indicating the quality of chest compression and a statistically significant better total compression score was obtained in comparison with non-real time monitoring of chest compression $(\mathrm{p}=0.001)$. The depth of chest compression in the experimental and control group showed statistically significant differences (51 mm [IQR 48-57] vs. $40 \mathrm{~mm}$ [IQR 39-44]; $\mathrm{p}<0.001$ ) respectively. Chest compression rate for the experimental group was 110 (IQR 103-121) CPM, and for the control group 124 (IQR 110-128 CPM; p = 0.019). Resuscitation 


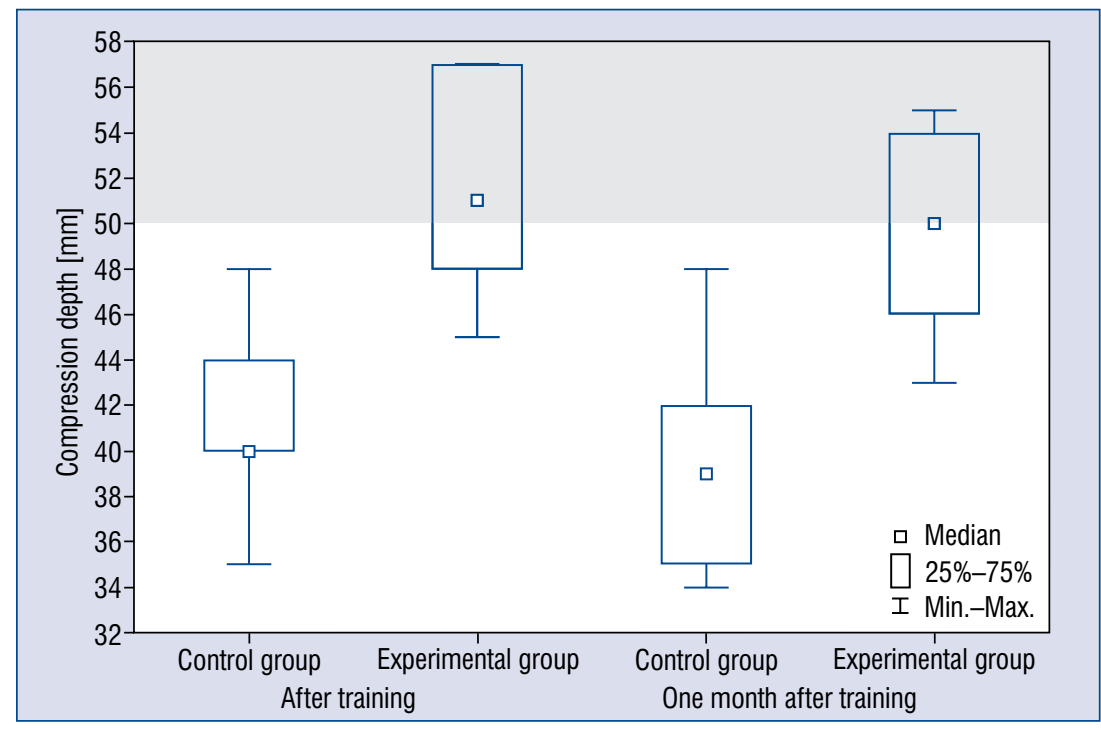

Figure 1. Median compression depth.

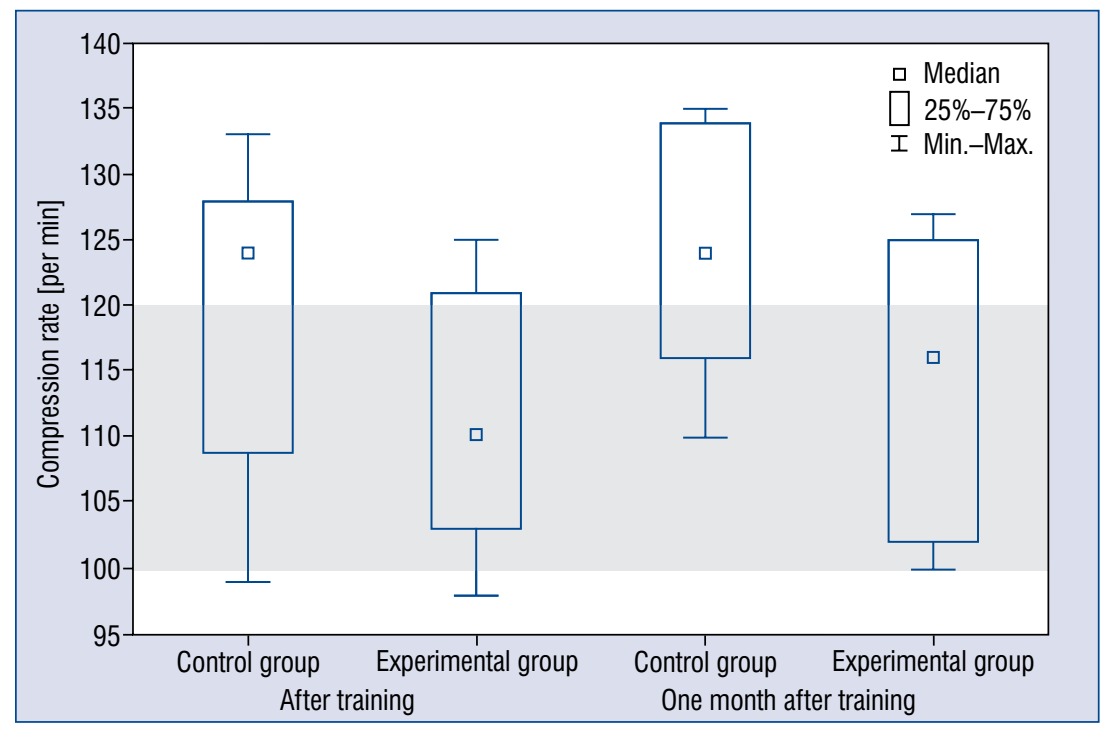

Figure 2. Median compression rate.

with a possibility to observe chest compression parameters was associated with better chest relaxation and better hand positioning (Table 1 ).

In the second phase of the study (1 month after the training) the depth of chest compressions in the experimental and control group was different and was $50 \mathrm{~mm}$ (IQR 46-54) vs. $39 \mathrm{~mm}$ (IQR $35-42 ; \mathrm{p}=0.001$; Fig. 1$)$. The chest compression rate achieved was 116 CPM (IQR 102-125) for the experimental group and 124 CPM (IQR 116-134; $\mathrm{p}=0.034$; Fig. 2) for the control group. The correctness of chest relaxation in the experimental group was $86 \%$ (IQR 68-89) and a statistically significant higher measure than in the control group - 74\% (IQR 47-80; $p=0.031$; Fig. 3).

The correct hand positioning, as well as compression depth compliance, compression rate compliance, and total compression score were significantly better statistically than in the experimental group in comparison with the control group ( $p<0.05$ for all parameters).

\section{Discussion}

The present study showed the validity of using systems which indicate the quality of chest 


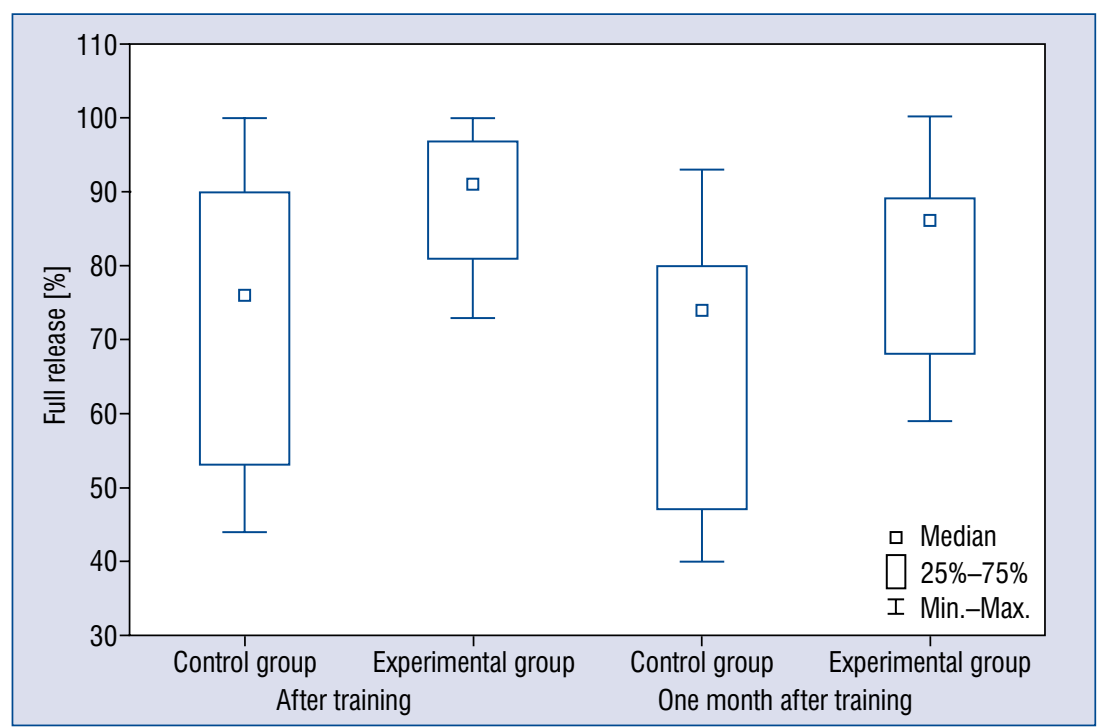

Figure 3. Median full release.

compression during teaching of basic resuscitation procedures because the correction in real time of the chest quality performed significantly improves overall quality of chest compression. Evaluation of chest compression quality with and without chest compression indicating software showed that subjects adjust to chest compression parameters in real time, and had significantly better results for all analyzed parameters compared to the group that could not observe the quality of their resuscitation.

The depth of chest compressions performed by the experimental group (with the possibility to assess the quality of compression in real time) was $51 \mathrm{~mm}$, while in the case of groups without this possibility $-40 \mathrm{~mm}(\mathrm{p}<0.001)$. According to ERC and AHA guidelines, the depth of chest compression in adults should be between 50 and $60 \mathrm{~mm}$ [20]. Numerous studies indicate an improvement in the quality of chest compressions when using CPR feedback devices, including TrueCPR, PocketCPR, CPRMeter or EasyCPR [21-23].

Another parameter indicated in the resuscitation guidelines as important for the quality of chest compression is the rate of chest compressions, which should be between 100 and 120 CPM [24]. In this post-training study, the rate of chest compressions was $124 \mathrm{CPM}$ for the control group and 110 CPM for the experimental group. During the evaluation phase of the study, 1 month after the training, the rates were 124 vs. $116 \mathrm{CPM}$, respectively. Jäntti et al. [25] as well as other authors' studies [13, 26,27] also indicate that manual chest compression is performed too rapidly. As Solevåg and Schmölzer [28] had indicated a rate higher than $120 / \mathrm{min}$ is also more fatiguing, which affects chest compression quality. On the other hand, Zou et al. [29] studies indicate that the optimal rate of chest compression is $120 / \mathrm{min}$. Studies published by Lee et al. [30] also indicate $120 \mathrm{CPM}$ as the optimal chest compression rate, while noting that higher compression rates can reduce chest relaxation. Similar conclusions can also be drawn from studies by Smereka et al. [8], as well as from studies by other authors [31-33].

Another equally important parameter is the correctness of chest relaxation. It is the compression of the chest to the appropriate depth and then allowing it to return to its normal shape before compression determines the appropriate difference in pressure in the chest to generate organ perfusion [5]. In a study conducted both immediately after the training and a month after the training, a higher percentage of correctly performed relaxation was obtained by participants from the experimental group who had the opportunity to observe the parameters of chest compression in real time during the training.

The use of a system that indicates, in real time, the quality of resuscitation during basic life support learning has allowed participants to improve chest compression parameters and could therefore have a real impact on a patient's chances of survival. An important conclusion from the results is that those who have learned resuscitation using monitoring software perform higher quality chest compressions 1 month subsequent to training. This 
may indicate a higher level of familiarity with this important skill of chest compression.

\section{Limitations of the study}

A limitation in this study is the use of medical simulation in the research process, however, this fact was intended and dictated by the fact that only during medical simulation was it possible to conduct such a study without potential harm to the patient [34]. An advantage of the study, in turn, is its randomized multi-center design, a relatively large study group, as well as undertaking an evaluation of chest compression skills not only immediately after training, but also 1 month after training.

\section{Conclusions}

Observing real-time chest compression quality parameters during BLS training may improve the quality of chest compression 1 month after training including correct hand positioning, compression depth and rate compliance.

\section{Acknowledgements}

The authors want to thank all participants for their participation in this study.

\section{Funding}

Study supported by the ERC Research Net, and the Polish Society of Disaster Medicine.

\section{Conflict of interest: None declared}

\section{References}

1. Gräsner JT, Bossaert L. Epidemiology and management of cardiac arrest: what registries are revealing. Best Pract Res Clin Anaesthesiol. 2013; 27(3): 293-306, doi: 10.1016/j.bpa.2013.07.008, indexed in Pubmed: 24054508.

2. Berdowski J, Berg RA, Tijssen JGP, et al. Global incidences of out-of-hospital cardiac arrest and survival rates: Systematic review of 67 prospective studies. Resuscitation. 2010; 81(11): 1479-1487, doi: 10.1016/j.resuscitation.2010.08.006, indexed in Pubmed: 20828914.

3. Jagosz A, Bursy D, Sobon A, et al. In-hospital sudden cardiac arrest protocol analysis. Kardiol Pol. 2018; 76(2): 376-380, doi: 10.5603/KP.a2017.0209, indexed in Pubmed: 29131292.

4. Vaillancourt C, Verma A, Trickett J, et al. Evaluating the effectiveness of dispatch-assisted cardiopulmonary resuscitation instructions. Acad Emerg Med. 2007; 14(10): 877-883, doi: 10.1197/j.aem.2007.06.021, indexed in Pubmed: 17761545.

5. Smereka J, Szarpak L, Rodríguez-Núñez A, et al. A randomized comparison of three chest compression techniques and associated hemodynamic effect during infant CPR: A randomized manikin study. Am J Emerg Med. 2017; 35(10): 1420-1425, doi: 10.1016/j.ajem.2017.04.024, indexed in Pubmed: 28433454.
6. Smereka J, Madziala M, Szarpak L. Comparison of two infant chest compression techniques during simulated newborn cardiopulmonary resuscitation performed by a single rescuer: a randomized, crossover multicenter trial. Cardiol J. 2018 [Epub ahead of print], doi: 10.5603/CJ.a2018.0090, indexed in Pubmed: 30155866.

7. Ewy GA, Zuercher M, Hilwig RW, et al. Improved neurological outcome with continuous chest compressions compared with 30:2 compressions-to-ventilations cardiopulmonary resuscitation in a realistic swine model of out-of-hospital cardiac arrest. Circulation. 2007; 116(22): 2525-2530, doi: 10.1161/CIRCULATIONAHA.107.711820, indexed in Pubmed: 17998457.

8. Smereka J, Iskrzycki $Ł$, Makomaska-Szaroszyk E, et al. The effect of chest compression frequency on the quality of resuscitation by lifeguards. A prospective randomized crossover multicenter simulation trial. Cardiol J. 2018 [Epub ahead of print], doi: 10.5603/CJ.a2018.0121, indexed in Pubmed: 30338845.

9. Czekjalo M. Are we prepared to use bag-mask-ventilation during continuous chest compression? Disaster and Emergency Medicine Journal. 2018; 3(4): 148-149, doi: 10.5603/demj.2018.0030.

10. Majer J, Madziala A, Dabrowska A, et al. The place of TrueCPR feedback device in cardiopulmonary resuscitation. Should we use it? A randomized pilot study. Disaster and Emergency Medicine Journal. 2018; 3(4): 131-136, doi: 10.5603/demj.2018.0028.

11. Jorge-Soto C, Abilleira-González M, Otero-Agra M, et al. Schoolteachers as candidates to be basic life support trainers: A simulation trial. Cardiol J. 2018 [Epub ahead of print], doi: 10.5603/ CJ.a2018.0073, indexed in Pubmed: 30009374.

12. Wieczorek W, Smereka J, Szarpak L, et al. Which position for resuscitation should we take? A randomized crossover manikin study. Am J Emerg Med. 2018; 36(5): 899-900, doi: 10.1016/j. ajem.2017.09.043, indexed in Pubmed: 29100786.

13. Szarpak L, Filipiak KJ, Ładny JR, et al. Should nurses use mechanical chest compression devices during CPR? Am J Emerg Med. 2016; 34(10): 2044-2045, doi: 10.1016/j.ajem.2016.07.057, indexed in Pubmed: 27528048.

14. Szarpak L, Truszewski Z, Czyzewski L, et al. CPR using the lifeline ARM mechanical chest compression device: a randomized, crossover, manikin trial. Am J Emerg Med. 2017; 35(1): 96-100, doi: 10.1016/j.ajem.2016.10.012, indexed in Pubmed: 27756513.

15. Kleinman ME, Goldberger ZD, Rea T, et al. 2017 American Heart Association Focused Update on Adult Basic Life Support and Cardiopulmonary Resuscitation Quality: An Update to the American Heart Association Guidelines for Cardiopulmonary Resuscitation and Emergency Cardiovascular Care. Circulation. 2018; 137(1): e7-ee13, doi: 10.1161/CIR.0000000000000539, indexed in Pubmed: 29114008.

16. Wieczorek W, Smereka J, Ladny JR, et al. The impact of a CPRezy ${ }^{\mathrm{TM}}$ feedback device on the quality of chest compressions performed by nurses. Am J Emerg Med. 2018; 36(7): 1318-1319, doi: 10.1016/j.ajem.2017.11.061, indexed in Pubmed: 29196113.

17. Kurowski A, Szarpak Ł, Bogdański $Ł$, et al. Comparison of the effectiveness of cardiopulmonary resuscitation with standard manual chest compressions and the use of TrueCPR and PocketCPR feedback devices. Kardiol Pol. 2015; 73(10): 924-930, doi: 10.5603/KP.a2015.0084, indexed in Pubmed: 25985725.

18. Oh JeH, Kim CW. The use of the PocketCPR application in basic life support training. Am J Emerg Med. 2017; 35(1): 189-190, doi: 10.1016/j.ajem.2016.10.082, indexed in Pubmed: 27839843.

19. Kleinman ME, Brennan EE, Goldberger ZD, et al. Part 5: Adult Basic Life Support and Cardiopulmonary Resuscitation Quality: 
2015 American Heart Association Guidelines Update for Cardiopulmonary Resuscitation and Emergency Cardiovascular Care. Circulation. 2015; 132(18 Suppl 2): S414-S435, doi: 10.1161/ CIR.0000000000000259, indexed in Pubmed: 26472993.

20. Monsieurs KG, Nolan JP, Bossaert LL, et al. ERC Guidelines 2015 Writing Group. European Resuscitation Council Guidelines for Resuscitation 2015: Section 1. Executive summary. Resuscitation. 2015; 95: 1-80, doi: 10.1016/j.resuscitation.2015.07.038, indexed in Pubmed: 26477410.

21. Truszewski Z, Szarpak L, Kurowski A, et al. Randomized trial of the chest compressions effectiveness comparing 3 feedback CPR devices and standard basic life support by nurses. Am J Emerg Med. 2016; 34(3): 381-385, doi: 10.1016/j.ajem.2015.11.003, indexed in Pubmed: 26612703.

22. Majer J, Kaminska H, Wieczorek W, et al. Impact of a CPRmeter feedback device on chest compression quality performed by nurses - a randomized crossover study. Disaster Emerg Med J. 2018; 3(1): 36-37, doi: 10.5603/demj.2018.0008.

23. Kurowski A, Czyżewski L, Bogdański L, et al. Quality of chest compression with CardioPump CPR compared to single rescuer standard BLS. Am J Emerg Med. 2015; 33(1): 114-115, doi: 10.1016/j.ajem.2014.10.027, indexed in Pubmed: 25455057.

24. Abelairas-Gómez C, Barcala-Furelos R, Szarpak $€$, et al. The effect of strength training on quality of prolonged basic cardiopulmonary resuscitation. Kardiol Pol. 2017; 75(1): 21-27, doi: 10.5603/KP.a2016.0165, indexed in Pubmed: 27878801.

25. Jäntti H, Silfvast T, Turpeinen A, et al. Influence of chest compression rate guidance on the quality of cardiopulmonary resuscitation performed on manikins. Resuscitation. 2009; 80(4): 453-457, doi: 10.1016/j.resuscitation.2009.01.001, indexed in Pubmed: 19203821.

26. Szarpak $€$, Truszewski Z, Smereka J, et al. Does the use of a chest compression system in children improve the effectiveness of chest compressions? A randomised crossover simulation pilot study. Kardiol Pol. 2016; 74(12): 1499-1504, doi: 10.5603/ KP.a2016.0107, indexed in Pubmed: 27391911.
27. Smereka J, Kasiński M, Smereka A, et al. The quality of a newly developed infant chest compression method applied by paramedics: a randomised crossover manikin trial. Kardiol Pol. 2017; 75(6): 589-595, doi: 10.5603/KP.a2017.0015, indexed in Pubmed: 28150278.

28. Solevåg AL, Schmölzer GM. Optimal Chest Compression Rate and Compression to Ventilation Ratio in Delivery Room Resuscitation: Evidence from Newborn Piglets and Neonatal Manikins. Front Pediatr. 2017; 5: 3, doi: 10.3389/fped.2017.00003, indexed in Pubmed: 28168185.

29. Zou Y, Shi W, Zhu Y, et al. Rate at 120 /min provides qualified chest compression during cardiopulmonary resuscitation. Am J Emerg Med. 2015; 33(4): 535-538, doi: 10.1016/j.ajem.2015.01.024, indexed in Pubmed: 25662803.

30. Lee SH, Ryu JiHo, Min MKi, et al. Optimal chest compression rate in cardiopulmonary resuscitation: a prospective, randomized crossover study using a manikin model. Eur J Emerg Med. 2016; 23(4): 253-257, doi: 10.1097/MEJ.0000000000000249, indexed in Pubmed: 25710082.

31. Idris AH, Guffey D, Aufderheide TP, et al. Resuscitation Outcomes Consortium (ROC) Investigators. Relationship between chest compression rates and outcomes from cardiac arrest. Circulation. 2012; 125(24): 3004-3012, doi: 10.1161/CIRCULATIONAHA.111.059535, indexed in Pubmed: 22623717.

32. Dosman CF, Jones RL. High-frequency chest compression: a summary of the literature. Can Respir J. 2005; 12(1): 37-41, doi: 10.1155/2005/525813, indexed in Pubmed: 15776126.

33. Austin AL, Spalding CN, Landa KN, et al. A Randomized Control Trial of Cardiopulmonary Feedback Devices and Their Impact on Infant Chest Compression Quality: A Simulation Study. Pediatr Emerg Care. 2017 [Epub ahead of print], doi: 10.1097/ PEC.0000000000001312, indexed in Pubmed: 29084067.

34. Smereka J, Szarpak L, Smereka A, et al. Evaluation of new two-thumb chest compression technique for infant CPR performed by novice physicians. A randomized, crossover, manikin trial. Am J Emerg Med. 2017; 35(4): 604-609, doi: 10.1016/j. ajem.2016.12.045, indexed in Pubmed: 28040386. 